\title{
Structure and far-infrared edge modes of quantum antidots at zero magnetic field
}

\author{
A. Emperador, M. Pi, and M. Barranco \\ Departament d'Estructura i Constituents de la Matèria, Facultat de Física, Universitat de Barcelona, E-08028 Barcelona, Spain \\ E. Lipparini \\ Dipartimento di Fisica, Università di Trento, 38050 Povo, Italy \\ L1. Serra \\ Departament de Física, Facultat de Ciències, Universitat de les Illes Balears, E-07071 Palma de Mallorca, Spain
}

(Received 10 February 1998)

\begin{abstract}
We have investigated edge modes of different multipolarity sustained by quantum antidots at zero magnetic field. The ground state of the antidot is described within a local-density-functional formalism. Two sum rules, which are exact within this formalism, have been derived and used to evaluate the energy of edge collective modes as a function of the surface density and the size of the antidot. [S0163-1829(98)00332-4]
\end{abstract}

With the progress of microstructure technology, the study of the two-dimensional electron gas (2DEG) has evolved to that of laterally confined superlattices made of either electron islands (dots) or holes surrounded by electrons (antidots). Much effort has been devoted in the past to the study of quantum dots, as compared to that put in the study of quantum antidots. One of the goals of their study has been to determine the far-infrared response of these semiconductor microstructures, and the formation of compressible and incompressible states when a magnetic field $B$ is perpendicularly applied. In the case of antidots, which is the subject of the present work, experimental evidence of collective excitations sustained by these structures has been presented in Refs. 1-3. A theoretical description based on magnetoplasmons in two-dimensional antidot structures has been given in Ref. 4 that compares well with the experimental data of Ref. 2. Recently, the existence of compressible and incompressible strips at the edge of antidots has been determined by far-infrared spectroscopy. ${ }^{5}$

We have started a systematic study of the structure and collective far-infrared response of antidots, whose aim is to achieve a level of sophistication in the description of these systems similar to that attained for quantum dots. As a first step, we present here results at zero magnetic field obtained within density-functional theory (DFT). To some extent, the present study is similar in scope to that carried out in Ref. 6 on the surface excitations of cavities in 3D metals. The $B \neq 0$ case, which requires a rather different and far more complex approach, will be presented in a forthcoming paper.

We have modeled an antidot of radius $R$ in a 2DEG of surface density $n_{s}$ by a positive jellium background of density $n_{s} \Theta(r-R)$ to which we have added a parabolic potential barrier of the type $V_{p}(r)=m \omega_{0}^{2}\left(R^{2}-r^{2}\right) / 2$ acting on the electrons for $r \leqslant R$. A potential barrier of one kind or another is needed to prevent the electrons from spilling in the antidot too much, producing an unphysical representation of the experimental device. Actually, we have found that if $\omega_{0}$ is set to zero, high-density antidots such as those studied in Ref. 5 would have nonzero electron densities even at $r=0$. We shall call $V_{\text {ext }}$ the sum of the jellium and $V_{p}$ potentials.
The ground state (gs) of the antidot is obtained solving the Kohn-Sham (KS) equations as indicated, for example, in Ref. 7. The problem is simplified by the imposed circular symmetry, and only the radial KS equations have to be considered to determine the electronic radial wave functions $R_{l}(r)$. We have used a dielectric constant $\epsilon$ and an electron effective mass $m=m^{*} m_{e}$, which are characteristics of GaAs: $\epsilon=12.4$ and $m^{*}=0.067$, which we have chosen for the numerical applications in view of the existing experimental data of Refs. 2 and 5. The single electron potential $V(r)$ entering the KS equations is made of $V_{\text {ext }}$, of the Hartree electron-electron potential and of the exchange-correlation potential. The correlation potential has been obtained from the correlation energy of Ref. 8 in a local-density approximation. Sometimes we shall use effective atomic units. In this system of units, the length unit is the Bohr radius times $\epsilon / m^{*}$, and the energy unit is the Hartree times $m^{*} / \epsilon^{2}$, denoted here as $a_{0}^{*}$ and $H^{*}$, respectively. For GaAs we have $a_{0}^{*} \sim 97.9 \AA$ and $H^{*} \sim 11.9 \mathrm{meV} \sim 95.6 \mathrm{~cm}^{-1}$.

Physically acceptable solutions to the radial KS equations have to be regular at $r=0$ and behave asymptotically as $R_{l}(r, k) \sim J_{l}(k r)+\tan \left(\delta_{l}\right) Y_{l}(k r)$, where $J_{l}$ and $Y_{l}$ are the integer Bessel functions of the first and second kind, ${ }^{9}$ and $k=\sqrt{2 m\left(E-V_{\infty}\right) / \hbar^{2}}$. The wave number $k$ has to be $k \leqslant k_{F}=\sqrt{2 \pi n_{s}}$. Taking into consideration the spin degeneracy, the electron density $\rho(r)$ is obtained as

$$
\rho(r)=\sum_{l=-\infty}^{l=\infty} \frac{2}{(2 \pi)^{2}} \int_{|\vec{k}| \leqslant k_{F}} R_{l}^{2}(r, k) d \vec{k}
$$

We have checked that the number of points used to carry out a Gaussian integration over $k$, and the maximum $|l| \mathrm{em}$ ployed in Eq. (1) lead to stable results.

We show in Fig. 1 the electronic densities corresponding to antidots of $R=7.5 a_{0}^{*}$ and $n_{s}=0.05,0.1,0.2,0.3$, and 0.4 $\left(a_{0}^{*}\right)^{-2}$. They span the size and density range of those fabricated and experimentally studied in Ref. 2, and have been obtained using $\omega_{0}=0.3 H^{*}$. 


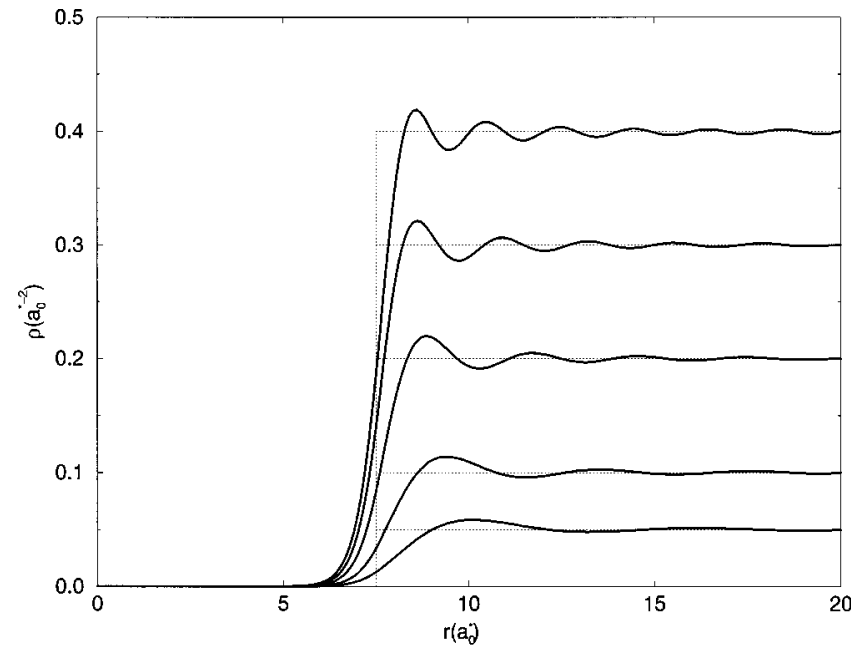

FIG. 1. Electronic densities as a function of $r$ for antidots of $R=7.5 a_{0}^{*}$ and $n_{s}=0.05,0.1,0.2,0.3$, and $0.4\left(a_{0}^{*}\right)^{-2}$. Also shown are the jellium densities (dotted lines).

These results can be employed to determine the $B=0$ far-infrared multipole response of antidots. To do so, we rely on the formalism described in detail in Ref. 10. For the present purpose, it consists in obtaining the so-called $m_{1}$ and $m_{3}$ sum rules (SR) for an excitation operator $Q_{L}$. We have that $^{11}$

$$
\begin{gathered}
m_{1}\left(Q_{L}\right)=\frac{1}{2}\left\langle 0\left|\left[Q_{L}^{+},\left[H, Q_{L}\right]\right]\right| 0\right\rangle, \\
m_{3}\left(Q_{L}\right)=\frac{1}{2}\left\langle 0\left|\left[\left[H,\left[H, Q_{L}^{+}\right]\right],\left[H, Q_{L}\right]\right]\right| 0\right\rangle,
\end{gathered}
$$

where $|0\rangle$ is the gs of the system. These SR have been extensively studied in the literature. ${ }^{11,12}$ As indicated in these references, if only a collective state is contributing to the strength function, $E_{3}\left(Q_{L}\right) \equiv\left(m_{3} / m_{1}\right)^{1 / 2}$ represents the average excitation energy. This is the situation experimentally found for antidots at zero magnetic field.

The operator $Q_{L}$ is taken to be

$$
Q_{L}=\sum_{j=1}^{N} \frac{1}{r_{j}^{L}} e^{i L \theta_{j}}
$$

This choice is inspired in that $(q r)^{-L} e^{i L \theta}$ is the small $q$ expansion of the function $Y_{L}(q r) e^{i L \theta}$, which is the restriction to the $z=0$ plane of the irregular solution of the Laplace equation in cylindrical coordinates. Following Ref. 10, a lengthy but straightforward calculation yields

$$
\begin{gathered}
m_{1}\left(Q_{L}\right)=2 \pi L^{2} \int_{0}^{\infty} d r \frac{1}{r^{2 L+1}} \rho(r), \\
m_{3}\left(Q_{L}\right)=m_{3}(T)+m_{3}(e e)+m_{3}\left(V_{e x t} e\right),
\end{gathered}
$$

where

$$
\begin{gathered}
m_{3}(T)=2 \pi L^{2}(L+1) \int_{0}^{\infty} d r \frac{1}{r^{2 L+3}}[L \tau(r)+2(L+2) \lambda(r)], \\
m_{3}(e e)=4 \pi L^{2} \frac{(2 L-1) ! !}{2^{L} L !} \int_{0}^{\infty} \rho^{\prime}(r) d r\left\{\frac{1}{r^{2 L+1}} \int_{0}^{r}\left[2 \rho^{\prime}\left(r^{\prime}\right)+r^{\prime} \rho^{\prime \prime}\left(r^{\prime}\right)\right] E_{L}\left(\frac{r^{\prime}}{r}\right) d r^{\prime}\right. \\
\left.+\int_{r}^{\infty} \frac{1}{r^{\prime(2 L+1)}}\left[(2 L+1) \rho^{\prime}\left(r^{\prime}\right)-r^{\prime} \rho^{\prime \prime}\left(r^{\prime}\right)\right] E_{L}\left(\frac{r}{r^{\prime}}\right) d r^{\prime}-\frac{2^{L+1} L !}{(2 L+1) ! !} \frac{1}{r^{2 L}} \rho^{\prime}(r)\right\} \\
+4 \pi L^{2} \int_{0}^{\infty} d r \frac{1}{r^{2 L+2}}\left[r \rho^{\prime \prime}(r)-(2 L+1) \rho^{\prime}(r)\right]\left\{\frac{1}{r} \int_{0}^{r}\left[3 r^{\prime} \rho\left(r^{\prime}\right)+r^{\prime} \rho^{\prime}\left(r^{\prime}\right)\right] \mathbf{E}\left(\frac{r^{\prime}}{r}\right) d r^{\prime}\right. \\
\left.-\int_{r}^{\infty} r^{\prime} \rho^{\prime}\left(r^{\prime}\right) \mathbf{E}\left(\frac{r}{r^{\prime}}\right) d r^{\prime}-2 r \rho(r)+\lim _{\infty \infty} R_{\infty} n_{s} \mathbf{E}\left(\frac{r}{R_{\infty}}\right)\right\}, \\
m_{3}\left(V_{e x t} e\right)=\pi L^{2} \int_{0}^{\infty} d r V_{e x t}(r)\left[\rho^{\prime \prime}(r)-\frac{2 L+1}{r} \rho^{\prime}\right] \frac{1}{r^{2 L+1}} .
\end{gathered}
$$

The definition of the densities $\tau(r)$ and $\lambda(r)$ and of the function $E_{L}$ can be found in Ref. 10, and the primes on the density denote $r$ derivatives. The jellium potential $V_{+}(r)$ entering in $V_{\text {ext }}$ is

$$
V_{+}(r)=4 n_{s} \begin{cases}R_{\infty} \mathbf{E}\left(r / R_{\infty}\right)-R \mathbf{E}(r / R), & r<R \\ R_{\infty} \mathbf{E}\left(r / R_{\infty}\right)-r \mathbf{E}(R / r)+r\left(1-(R / r)^{2}\right) \mathbf{K}(R / r), & r>R .\end{cases}
$$




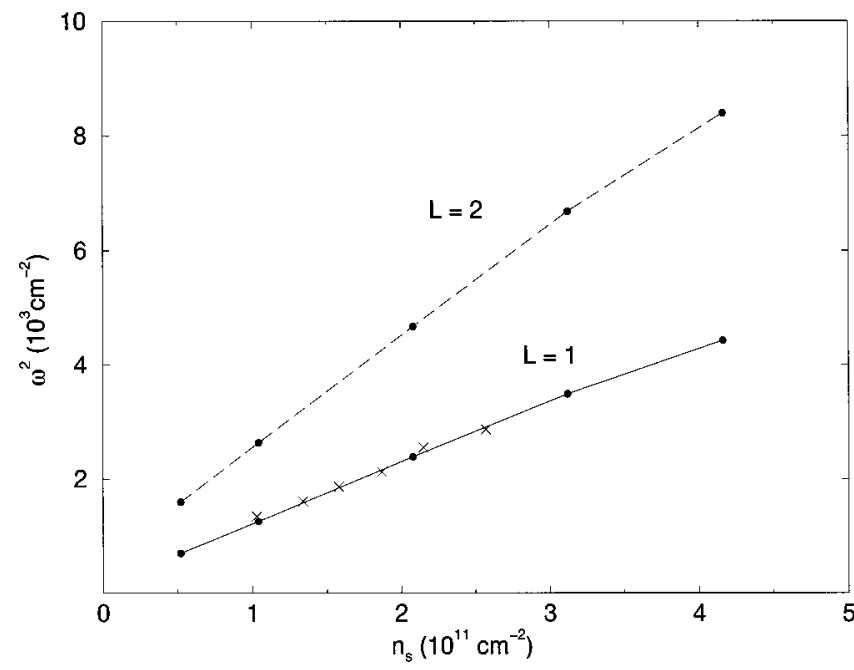

FIG. 2. Mode frequency for $L=1$ and 2 as a function of the electron surface density corresponding to $R=7.5 a_{0}^{*}$. The crosses are experimental data from Ref. 2 , and the lines are drawn to guide the eye.

In the above equations, $\mathbf{K}$ and $\mathbf{E}$ are the complete elliptic integrals of the first and second kind, respectively, ${ }^{13}$ and $R_{\infty}$ represents a large $r$ value. In practice, it is the largest $r$ used in the structure calculation, which we have also taken as the point where the asymptotic behavior of $R_{l}(r)$ holds. We want to point out that the two Coulomb diverging terms in $m_{3}(e e)$ and $m_{3}\left(V_{e x t} e\right)$ cancel each other.

The present formalism can be applied to antidots with the restriction that $\rho(r)$ vanishes in a small disk around the origin. In practice, this is not a limitation, as can be inferred from Fig. 1 (see also Fig. 3). Some technical details about how the above integrals are handled can be found in Sec. IV of Ref. 6.

For a large antidot, the electronic density is constant everywhere apart from a narrow region along the border of the hole. Following the method outlined in Ref. 10, it is easy to show that $E_{3}$ yields the classical hydrodynamics dispersion relation for edge waves, namely, $E_{3}=\omega(q)$ $\sim \sqrt{2 n_{s} q \ln \left(q_{0} / q\right)}$. It is also worth to notice that the induced (or transition) density associated to the operator $Q_{L}$ has the form $^{10}$

$$
\rho_{t r}(\vec{r}) \propto L \frac{1}{r^{L+1}} \rho^{\prime}(r)
$$

that manifests the edge character of the excitation.

Figure 2 shows the frequency of the $L=1$ mode as a function of $n_{s}$ compared with the experimental points of Ref. 2 . For completeness, we have also plotted the results corresponding to $L=2$. We have checked that similar results are obtained using as potential barrier the parabola $V_{p}(r)$ $=m \omega_{0}^{2}(R-r)^{2} / 2$ for $r \leqslant R$ with $\omega_{0}=1 H^{*}$. One can see that the agreement with experiment is good. Furthermore, our calculation yields a frequency of $\sim 68 \mathrm{~cm}^{-1}$ for the $R$ $=180 \mathrm{~nm}, n_{s}=9 \times 10^{11} \mathrm{~cm}^{-2}$ antidot, in good agreement with the experimental findings of Ref. 5 .

If the electronic density $\rho(r)$ is approximated by a quasistep function, an analytical expression can be obtained for $E_{3}^{2}$. Proceeding as in Ref. 10 one gets

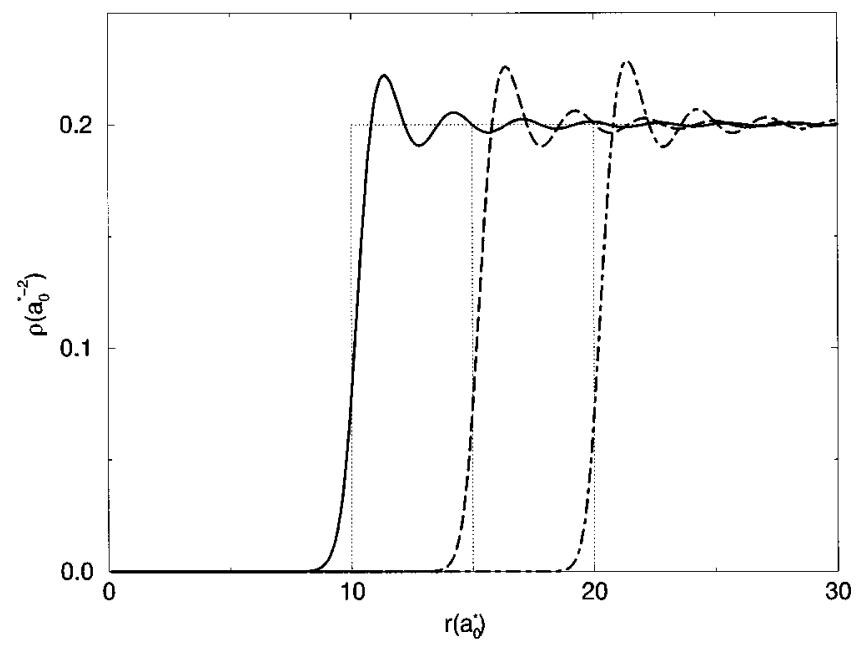

FIG. 3. Electronic densities as a function of $r$ for antidots of $R=10,15$, and $20 a_{0}^{*}$, and $n_{s}=0.2\left(a_{0}^{*}\right)^{-2}$. Also shown are the jellium densities (dotted lines).

$$
E_{3}^{2}=2 \pi n_{s} \frac{L(L+1)}{R^{2}}+4 n_{s} \frac{L}{R}\left[\frac{1}{2} \ln \left(\gamma \frac{R}{a}\right)+1-\sum_{m=1}^{L} \frac{1}{2 m-1}\right],
$$

where $a$ represents the width of the quasistep function, and the precise value of $\gamma$ depends on the way the electronic density goes to zero. ${ }^{14}$ This equation tells one that the frequencies have a $1 / \sqrt{R}$ linear dependence if the Coulomb energy contribution dominates. For reasonable values of $\gamma / a$, it happens for any realistic value of $R$.

We have also studied the size dependence of the mode energy. Figure 3 represents the electronic densities for antidots of $R=10,15$, and $20 a_{0}^{*}$, and a surface density $n_{s}=0.2\left(a_{0}^{*}\right)^{-2}$. The frequencies of the $L=1$ and 2 modes are shown in Fig. 4 as a function of $1 / \sqrt{R}$. They exhibit a distinct $R$ dependence, indicating a clear departure from parabolicity of the confining potential, i.e., a physical situation where the generalized Kohn theorem does not apply.

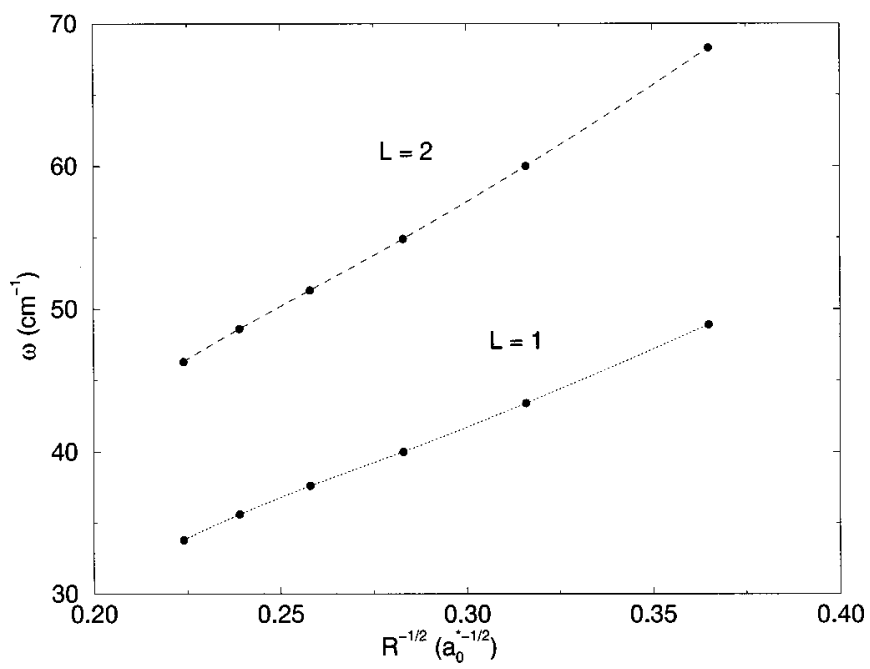

FIG. 4. $L=1$ and 2 mode frequencies as a function of $R^{-1 / 2}$ for $n_{s}=0.2\left(a_{0}^{*}\right)^{-2}$. From right to left, the dots correspond to $R=7.5,10,12.5,15,17.5$, and $20 a_{0}^{*}$. The lines are drawn to guide the eye. 
In conclusion, we have shown that density-functional theory is able to reproduce the zero-magnetic-field frequency of antidot edge modes in a way that is quite similar to that for quantum dots. Although a satisfactory description of the collective spectrum of antidots can be achieved using a magnetoplasmon approach, ${ }^{4}$ an alternative method based on a more microscopic approach such as DFT is needed to discuss other interesting problems, such as edge reconstruction and the formation of compressible and incompressible strips at the antidot edge. ${ }^{5}$

It is a pleasure to thank Ricardo Mayol and Francesc Salvat for useful discussions. This work has been performed under Grant Nos. PB95-1249 and PB95-0492 from CICYT, Spain, and GRQ94-1022 from Generalitat of Catalunya. A.E. acknowledges support from the Dirección General de Enseñanza Superior (Spain).
${ }^{1}$ K. Kern, D. Heitmann, P. Grambow, Y. H. Zhang, and K. Ploog, Phys. Rev. Lett. 66, 1618 (1991).

${ }^{2}$ Y. Zhao, D. C. Tsui, M. Santos, M. Shayegan, R. A. Ghanbari, D. A. Antoniadis, and H. I. Smith, Appl. Phys. Lett. 60, 1510 (1992).

${ }^{3}$ A. Lorke, I. Jejina, and J. P. Kotthaus, Phys. Rev. B 46, 12845 (1992).

${ }^{4}$ G. Y. Wu and Y. Zhao, Phys. Rev. Lett. 71, 2114 (1993).

${ }^{5}$ K. Bollweg, T. Kurth, D. Heitmann, V. Gudmundsson, E. Vasiliadou, P. Grambow, and K. Eberl, Phys. Rev. Lett. 76, 2774 (1996).

${ }^{6}$ L1. Serra, F. Garcias, J. Navarro, N. Barberán, M. Barranco, and M. Pi, Phys. Rev. B 46, 9369 (1992).
${ }^{7}$ M. Ferconi and G. Vignale, Phys. Rev. B 50, 14722 (1994).

${ }^{8}$ B. Tanatar and D. M. Ceperly, Phys. Rev. B 39, 5005 (1989).

${ }^{9}$ M. Abramowitz and I. A. Stegun, Handbook of Mathematical Functions (Dover, New York, 1970).

${ }^{10}$ E. Lipparini, N. Barberán, M. Barranco, M. Pi, and L1. Serra, Phys. Rev. B 56, 12375 (1997).

${ }^{11}$ E. Lipparini and S. Stringari, Phys. Rep. 175, 103 (1989).

${ }^{12}$ O. Bohigas, A. M. Lane, and J. Martorell, Phys. Rep. 51, 267 (1979).

${ }^{13}$ I. S. Gradshteyn and I. M. Ryzhik, Table of Integrals, Series and Products (Academic Press, New York, 1980).

${ }^{14}$ S. Giovanazzi, L. Pitaevskii, and S. Stringari, Phys. Rev. Lett. 72, 3230 (1994). 